> Composée de l'agencement de différents collagènes, protéoglycanes et glycoprotéines, la matrice extracellulaire constitue un substrat essentiel pour la morphogenèse tissulaire et procure soutien et flexibilité aux tissus matures. De plus, elle agit en tant qu'entité épigénétique d'information dans le sens où elle transmet et intègre des signaux extracellulaires par le biais de divers récepteurs de la membrane plasmique cellulaire. Parmi les molécules de la matrice extracellulaire présentes au cours du développement du système nerveux central (SNC), la ténascine-C (TN-C) possède un profil d'expression extrêmement particulier non seulement quant à sa distribution spatio-temporelle, mais, également, quant aux isoformes synthétisées. De nombreuses études réalisées in vitro ont suggéré des rôles spécifiques de la TN-C dans le développement du SNC, notamment dans la migration de précurseurs neuronaux ou dans la croissance et le guidage neuritiques. Aussi, l'invalidation du gène codant pour la TN-C chez la souris, initialement décrite sans phénotype structural apparent, a permis d'explorer plus en détail et in vivo l'implication potentielle de cette glycoprotéine dans l'ontogenèse du SNC. Les résultats récemment obtenus tendent à confirmer les travaux plus anciens mais aussi à révéler des fonctions nouvelles, en particulier dans la prolifération des précurseurs oligodendrocytaires. <

\title{
La ténascine-C: une molécule de la matrice extracellulaire impliquée dans le développement du système nerveux central
}

\author{
Emmanuel Garcion, Andreas Faissner, \\ Charles ffrench-Constant
}

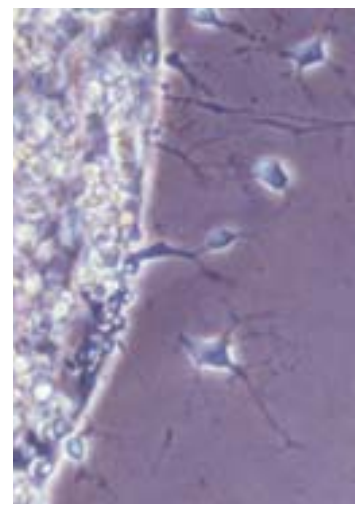

micro-environnement, telles que les facteurs de croissance et les molécules de la matrice extracellulaire, au nombre desquelles figure la TN-C. Successivement connue sous le nom d'antigène glio-mésenchymateux de la matrice extracellulaire (GMEM), d'antigène myotendineux, d'hexabrachion, de cytotactine, de J1 220/200, de ténascine et de neuronectine, la TN-C appartient à une famille de glycoprotéines oligomériques qui contient cinq membres connus, les ténascines $C, R, X, Y$ et $W$ [l]. En conditions natives et rapidement après le début de sa traduction, la $\mathrm{TN}-\mathrm{C}$ forme un hexamère résultant de la liaison de six monomères par des ponts disulfures $[1,2]$. Chaque monomère de $\mathrm{TN}-\mathrm{C}$ est constitué de différents domaines à structures répétées (Figure 1A). La forme monomérique de la TN-C est ainsi composée d'un minimum de huit modules de type III de la fibronectine (FNIII) auxquels peuvent être ajoutées par épissage alternatif d'autres répétitions de même type entre le cinquième et le sixième module (Figure 1). À partir de l'épissage de six modules chez la souris et de neuf chez l'homme, un nombre de combinai- 
sons de $2^{6}=64$ chez la souris et de $2^{9}=512$ chez l'homme peut théoriquement être obtenu, indiquant l'existence potentielle d'autant d'isoformes de TN-C. Toutefois, il a été démontré que seules certaines d'entre elles sont effectivement synthétisées dans le SNC [3]. Une disparition des formes longues de TN-C au profit des formes courtes a également été démontrée au cours du développement du SNC [3]. Ajoutant à la complexité de ces isoformes, une grande hétérogénéité de glycosylation de chacun des monomères de TN-C a été décrite, modifiant ainsi leur poids moléculaire apparent qui fluctue entre 190 et $240 \mathrm{kDa}$ chez la souris, et 180 et $320 \mathrm{kDa}$ chez l'homme [4]. Compte tenu de la variabilité de chaque monomère, la question est de savoir si l'assemblage en hexamère se fait exclusivement à partir de monomères identiques ou peut associer des monomères différents. Après un cheminement relativement lent du réticulum endoplasmique vers l'extérieur de la cellule, les hexamères de TN-C sont incorporés dans la matrice extracellulaire selon des mécanismes impliquant à la fois des protéines accessoires de la matrice extracellulaire et des récepteurs de la membrane plas-

$\mathbf{A}$

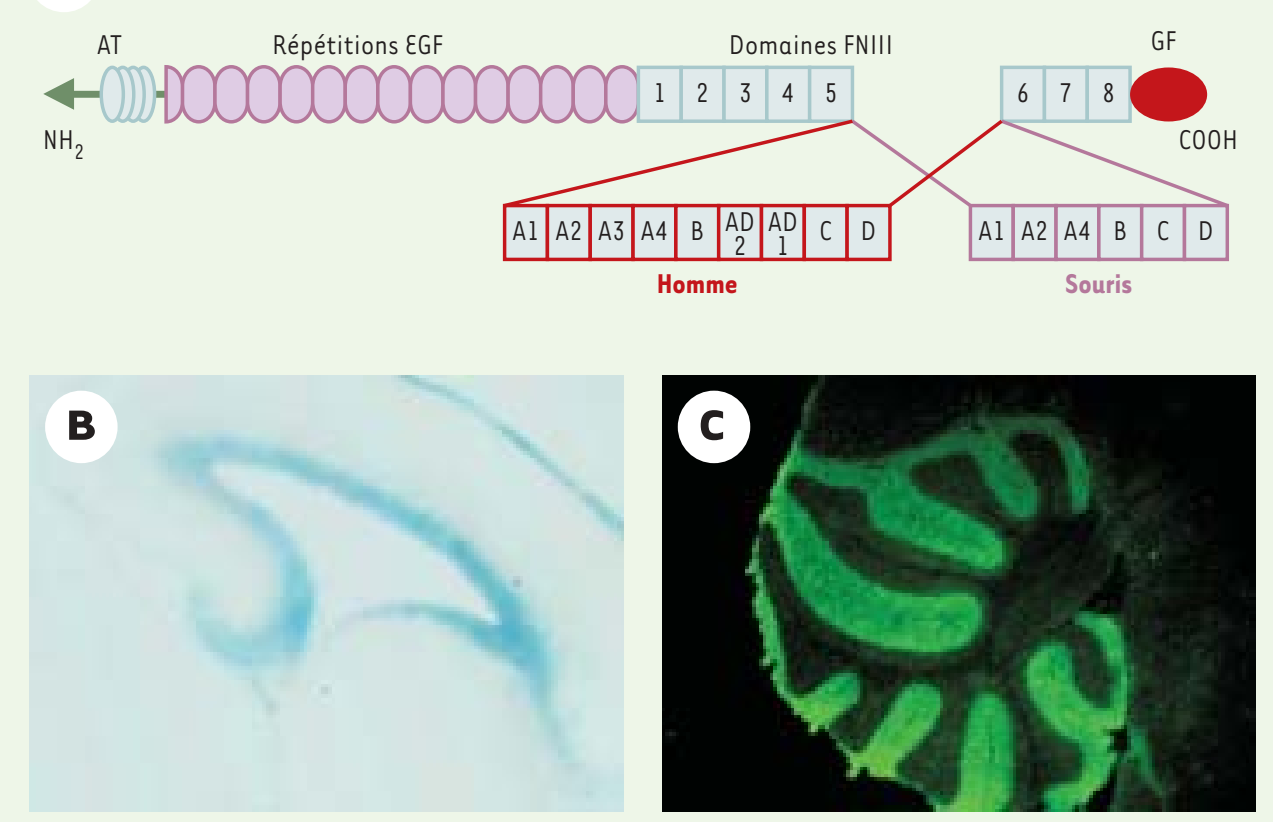

Figure 1. Structure et profil d'expression de la TN-C. A. Représentation schématique d'un monomère de TN-C comprenant un domaine $\mathrm{N}$-terminal (AT) riche en cystéines permettant l'assemblage en hexamère, suivi de 14,5 répétitions de type EGF (epidermal growth factor), puis de répétitions analogues aux modules de type III de la fibronectine (FNIII) dont certaines peuvent être alternativement épissées (chez l'homme et chez la souris), et, enfin, d'un globe $C$-terminal (GF) similaire aux chaînes $\beta$ et $\gamma$ du fibrinogène. $B$. Expression à $\varepsilon 17,5$ dans la zone sousventriculaire du transgène $L a c-Z$ en lieu et place de celle du gène de la TN-C chez des souris dont le gène de la TN-C a été invalidé. C. Détection immunohistologique de la TN-C dans la couche moléculaire du cervelet chez des souris témoins $\mathrm{Fl}$ (57BI/6) x CBA adultes. mique cellulaire [5]. La dégradation de la TN-C est, quant à elle, effectuée par divers membres de la famille des métalloprotéinases matricielles (MMP), capables de cliver les hexabrachions ${ }^{\star}$ en des sites spécifiques, et ayant pour effet, soit effectivement de dégrader la molécule, soit d'engendrer des formes monomériques à partir de l'hexamère natif $[1]$.

\section{Expression de la TN-C au cours du développement du SNC}

Présente lors de la gastrulation et lors de la formation des somites, la TN-C est exprimée très tôt au cours du développement [6]. Elle est synthétisée dans de nombreux tissus embryonnaires, notamment nerveux et conjonctifs, comme les crêtes neurales ou l'os. Sa distribution spatiotemporelle est précisément contrôlée et son expression spécifiquement associée à des structures particulières de l'organe au sein duquel elle est observée. Dans les tissus adultes, au contraire, la TN-C est peu exprimée, à l'exception des sites de remodelage et de migration cellulaires, des cicatrices tissulaires, à proximité de vaisseaux sanguins endommagés et au sein de nombreux types de tumeurs [5]. La production de TN-C dans le SNC murin a été mise en évidence dès le $10^{\mathrm{e}}$ jour de la vie embryonnaire ( $\varepsilon 10$ ) [7]. La TN-C est dès lors exprimée pendant toute l'ontogenèse du SNC de manière relativement étendue (cortex cérébral, striatum, capsule interne, noyaux hypothalamiques, noyau trigéminé, bulbe olfactif, cervelet et nerf optique). Elle est essentiellement synthétisée par les astrocytes immatures et réactifs, ainsi que par la glie radiaire et par la glie de Bergman [4, 7]. De plus, les précurseurs oligodendrocytaires sont capables, au moins in vitro, de produire des ARN messagers et certaines isoformes protéiques de la TN-C [8]. Absente du

* Molécule de forme hexamérique agencée en 6 bras.

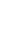


neuro-épithélium germinatif primaire, la TN-C n'est pas exprimée par les cellules souches nerveuses précoces (par exemple à $\varepsilon 8,5$ chez la souris). En revanche, de nombreux progéniteurs, issus de ce territoire initial et ne pouvant avec certitude être qualifiés de cellules souches in vivo, faute de marqueurs spécifiques, sont capables de produire l'hexabrachion, plus spécialement dans la zone sous-ventriculaire (Figure IB). Parallèlement, des sous-populations de neurones immatures, dont les cellules granulaires de l'hippocampe, certains neurones rétiniens ainsi que des motoneurones spinaux, synthétisent la TN-C $[4,7]$. Au fur et à mesure de la maturation du SNC, les niveaux d'expression de la TN-C se réduisent et sa production, chez l'adulte, n'est plus observée qu'au sein de régions particulières, incluant des sites de neurogenèse active tels que l'hippocampe, la zone sous-ventriculaire, la bordure de la voie de migration rostrale, ainsi que les zones dans lesquelles les axones ne sont pas myélinisés telles que la jonction entre le nerf optique et la rétine, et la couche moléculaire du cervelet (Figure 1C).

\section{Régulation de l'expression de la TN-C}

L'expression du gène codant pour la TN-C est sous le contrôle de signaux intracellulaires induits par divers facteurs solubles, des intégrines et le stress mécanique. Ainsi, le basic fibroblast growth factor (bFGF), le transforming growth factor $\beta$ l (TGF- $\beta \mathrm{l}$ ), le tumor necrosis factor $\alpha$ (TNF- $\alpha$ ), l'interleukine-1, le nerve growth factor (NGF) et le keratinocyte growth factor (KGF) sont autant d'inducteurs potentiels de la synthèse de TN-C [5]. Des signaux activés par les métalloprotéinases matricielles (notamment MMP 2) et des intégrines (notamment $\alpha v \beta 3$ ) peuvent également induire la production de TN-C [5]. Parallèlement, les forces mécaniques ont une importance primordiale dans la régula- tion de l'expression du gène codant pour la TN-C, et il apparaît que les intégrines peuvent jouer le rôle de capteurs de ces forces. En outre, les protéines Rho-GTPase, la F-actine et diverses kinases dont la MAPK (mitogen activated protein kinase) et la FAK (focal adhesion kinase), ont été impliquées comme intermédiaires de ce type de signaux [5]. Parmi les régulateurs transcriptionnels du gène codant pour la TN-C, la famille des protéines à homéodomaines joue un rôle essentiel [5]. L'analyse détaillée des interactions entre ces différents facteurs nucléaires et les éléments de réponse auxquels ils se lient reste à accomplir afin de déterminer leurs modalités d'action (induction, répression, synergie, opposition). Nombre de ces signaux présents au sein des tissus embryonnaires sont aussi présents dans les tissus adultes en cas de lésion, de remodelage, ou de processus tumoral, et permettent la ré-expression de la molécule dans des structures d'où elle avait disparu. Toutefois, les isoformes synthétisées dans ces cas diffèrent souvent de celles produites au cours du développement et il demeure essentiel, compte tenu de la spécificité de chacune d'entre elles pour leurs récepteurs, d'en déterminer la signification biologique.

\begin{tabular}{|c|c|c|}
\hline Familles moléculaires & TN-C récepteur/ligand & Domaines TN-C impliqués \\
\hline Intégrines & $\begin{array}{l}\alpha \times \beta 1 \\
\alpha 2 \beta 1 \\
\alpha v \beta 3 \\
\alpha 8 \beta 1 \\
\alpha 9 \beta 1\end{array}$ & $\begin{array}{l}\text { FNIII-3 } \\
\text { GF } \\
\text { FNIII-3 } \\
\text { GF } \\
\text { FNIII-3 } \\
\text { FNIII-3 }\end{array}$ \\
\hline $\lg$ CAM & Contactine Fll/F3 & FNIII-5-6 \\
\hline Facteurs de différenciation de type EGF & CALEB & GF \\
\hline $\begin{array}{l}\text { Protéines de liaison (dépendante du } \mathrm{Ca}^{2+} \text { ) } \\
\text { aux phospholipides membranaires }\end{array}$ & Annexine II & FNIII-A-D \\
\hline Récepteurs tyrosine kinases & $\varepsilon G F-R$ & $\varepsilon G F$ \\
\hline Récepteurs tyrosine phosphatases & RPTP- $\beta / \zeta$ & GF \\
\hline Canaux ioniques & $\begin{array}{l}\mathrm{Na}^{+} \text {-type II } \\
\text { dépendant du potentiel }\end{array}$ & $\begin{array}{l}\text { FNIII-1-2 } \\
\text { FNIII-A } \\
\text { FNIII-B } \\
\text { FNIII-6-8 }\end{array}$ \\
\hline $\begin{array}{l}\text { Composants de la matrice extracellulaire, } \\
\text { protéoglycanes et glycoprotéines }\end{array}$ & $\begin{array}{l}\text { Héparine } \\
\text { Phosphacane } \\
\text { Neurocane } \\
\text { Perlecane } \\
\text { Agrine } \\
\text { Syndécane } \\
\text { Glypicane } \\
\text { Fibronectine }\end{array}$ & $\begin{array}{l}\text { FNIII-3-5 } \\
\text { FNIII-5 } \\
\text { GF } \\
\text { GF } \\
\text { FNIII-4-5 } \\
\text { GF } \\
\text { FNIII-3-5 } \\
? \\
\text { GF } \\
\text { FNIII-5 } \\
\text { FNIII-6-8 }\end{array}$ \\
\hline
\end{tabular}

Tableau I. Récepteurs de la TN-C et domaines moléculaires impliqués. CAM : cell adhesion molecule ; CALEB : chicken acidic leucine-rich EGF-like domain containing brain protein. 


\section{Récepteurs et ligands de la TN-C}

Le nombre des récepteurs présomptifs de la TN-C ne cesse de croître au cours des années et les segments spécifiques de la molécule de $\mathrm{TN}-\mathrm{C}$ avec lesquelles ils interagissent sont progressivement déterminés [1] (Tableau 1). Parmi les récepteurs cellulaires de la TN-C, les intégrines représentent la famille moléculaire la plus importante. La TN-C s'associe également à de nombreux composants de la matrice extracellulaire, jouant un rôle important dans sa composition et son organisation. Cette pléiade de ligands laisse entrevoir un large spectre d'action de la TN-C sur le comportement cellulaire et il apparait que le répertoire de récepteurs d'un type cellulaire donné définit le champ d'activité biologique d'une isoforme donnée de TN-C.

\section{Implication fonctionnelle de la TN-C dans le développement neuronal}

Initialement connue pour ses propriétés anti-adhérentes, entraînant de multiples effets sur l'adhérence, la différenciation et la motilité cellulaires [1], la TN-C est manifestement douée de fonctions biologiques informatives propres par le biais des divers récepteurs auxquels elle se lie. Toutefois, la première fonction identifiée de la TN-C au sein du SNC fut celle de substrat répulsif pour des neurones embryonnaires mésencéphaliques et hippocampaux, ainsi que des neurones post-natals du cervelet pouvant choisir entre la TN-C et un substrat plus permissif à l'adhérence cellulaire [9]. In vitro, la TN-C entraîne aussi une déflexion du cône de croissance des neurites en faisant intervenir une réorganisation du cytosquelette [4]. Ces caractéristiques répulsives et inhibitrices de la TN-C sur l'attachement cellulaire peuvent être mises à l'actif de ses interférences avec la formation de points d'adhérence focale [5]. En outre, la TN-C est également capable d'activités proadhérentes pour les neurones lorsqu'ils sont cultivés sur un tapis astrocytaire [4]. Ces divergences d'effets ont conduit à analyser en détail les propriétés des différents domaines de la TN-C par des études fonctionnelles utilisant des anticorps monoclonaux spécifiques et des fragments recombinants de la molécule [10]. Ainsi, les répétitions de type EGF sont-elles en partie responsables des propriétés antiadhérentes de la $\mathrm{TN}-\mathrm{C}$, et le domaine FNIII-3 impliqué dans sa propriété d'inhiber l'attachement neuronal et la croissance neuritique; dans ce cas, les intégrines $\alpha v \beta 3$ et $\alpha 9 \beta 1$ (qui se lient au domaine FNIII-3 de la TN-C d'une manière respectivement dépendante et indépendante du tripeptide RGD) jouent le rôle de récepteurs. II a également été découvert que le domaine FNIII-B-D stimule la croissance axonale, ce que ne fait pas le domaine FNIII-Al-A2-A4 [4].
Ainsi, les hypothèses formulées quant au rôle de la TN-C dans la migration des cellules granulaires du cervelet [11] et dans l'innervation thalamo-corticale [12] ont été confirmées par tout ce travail d'identification moléculaire. Mais, curieusement, les souris déficientes pour le gène de la TN-C se développent, se reproduisent normalement, et n'arborent aucune anomalie structurale évidente [13, 14]. Néanmoins, des études spécifiques de ces mutants ont permis de mettre en évidence certains phénotypes plus subtils, touchant notamment le comportement locomoteur de ces souris [15, 16] et la neurochimie de leur cerveau, avec entre autres une diminution de l'activité de la tyrosine hydroxylase et de la production de neuropeptide $y$, alors que les niveaux de préprotachykinine et de cholécystokinine sont augmentés [16]. Enfin, une implication de la TN$C$ dans la synaptogenèse, et plus particulièrement dans le ciblage et l'induction des contacts synaptiques, est fortement suggérée [17, 18]. En accord avec ce concept, la TNC est induite dans les neurones granulaires du gyrus dentatus lors de potentialisation à long terme hippocampique et I'utilisation d'anticorps monoclonaux a permis de démontrer que le domaine FNIII-6-8 de la TN-C était impliqué dans l'apprentissage acoustique [18]. En périphérie, les souris dont le gène codant pour la TN-C a été invalidé présentent une perte de l'intégrité de la jonction neuro-musculaire et de certaines propriétés de ré-innervation. Cette observation renforce l'idée d'une implication fonctionnelle de la TN-C dans la stabilisation synaptique [19, 20].

\section{TN-C et comportement des précurseurs oligodendrocytaires}

Les précurseurs oligodendrocytaires, originaires de la zone ventriculaire de la moelle épinière et du télencéphale, suivent un schéma rigoureux de maturation comprenant leur migration, leur prolifération et leur différenciation terminale en oligodendrocytes myélinisants, phénomène ultime soumis à une sélection qui, pour environ $50 \%$ d'entre eux, se soldera par leur disparition par apoptose (Figure 2A). La distribution préférentielle de la TN-C dans les voies de migration des précurseurs oligodendrocytaires avait suggéré son rôle direct dans leur migration [21]. Aussi, le fait que la TN-C soit fortement exprimée dans des régions où les axones ne sont pas myélinisés - couche moléculaire du cervelet, portion proximale du nerf optique - a conduit à envisager sa responsabilité dans le profil final de myélinisation du SNC. L'analyse des souris transgéniques déficientes pour le gène de la TN-C montre qu'il n'en est rien et que la TN-C n'est responsable ni de l'absence de pénétration des précurseurs oligodendrocytaires dans la partie proximale du nerf optique et dans la couche moléculaire du 
cervelet (Figure 2D), ni du profil de distribution terminal des oligodendrocytes matures (Figure $2 \mathrm{~K}$ T) $[15,22]$. Si la TN-C ne constitue pas une barrière moléculaire, elle est en revanche fortement impliquée dans la migration de ces cellules. Des analyses in vitro ont démontré des propriétés inhibitrices spécifiques du substrat, de l'hexabrachion sur la migration des précurseurs oligodendrocytaires [23] et des propriétés antimigratoires intrinsèques de la molécule purifiée [8]. Ces effets de la TN-C impliquent au moins deux types d'inhibitions, l'une résultant de ses propriétés anti-adhérentes imputées aux répétitions de type EGF, et l'autre impliquant sa liaison à un ligand non encore identifié via le domaine FNIII-7-8 (Figures 1 et 3) [8]. In vivo, l'absence de TN-C entraîne une accélération de la migration des précurseurs oligodendrocytaires dans le nerf optique murin au cours du développement [22]. L'analyse de cet effet in vitro, en utilisant des matrices astrocytaires issues de cerveaux de souris témoins ou de cerveaux de souris dont le gène codant pour la TN-C a été invalidé, suggère un effet indirect de l'hexabrachion impliquant la présence d'un autre facteur dépendant de la TN-C (Figure 2 B, C, Figure 3) [22]. Parallèlement, nous avons démontré que la TN-C
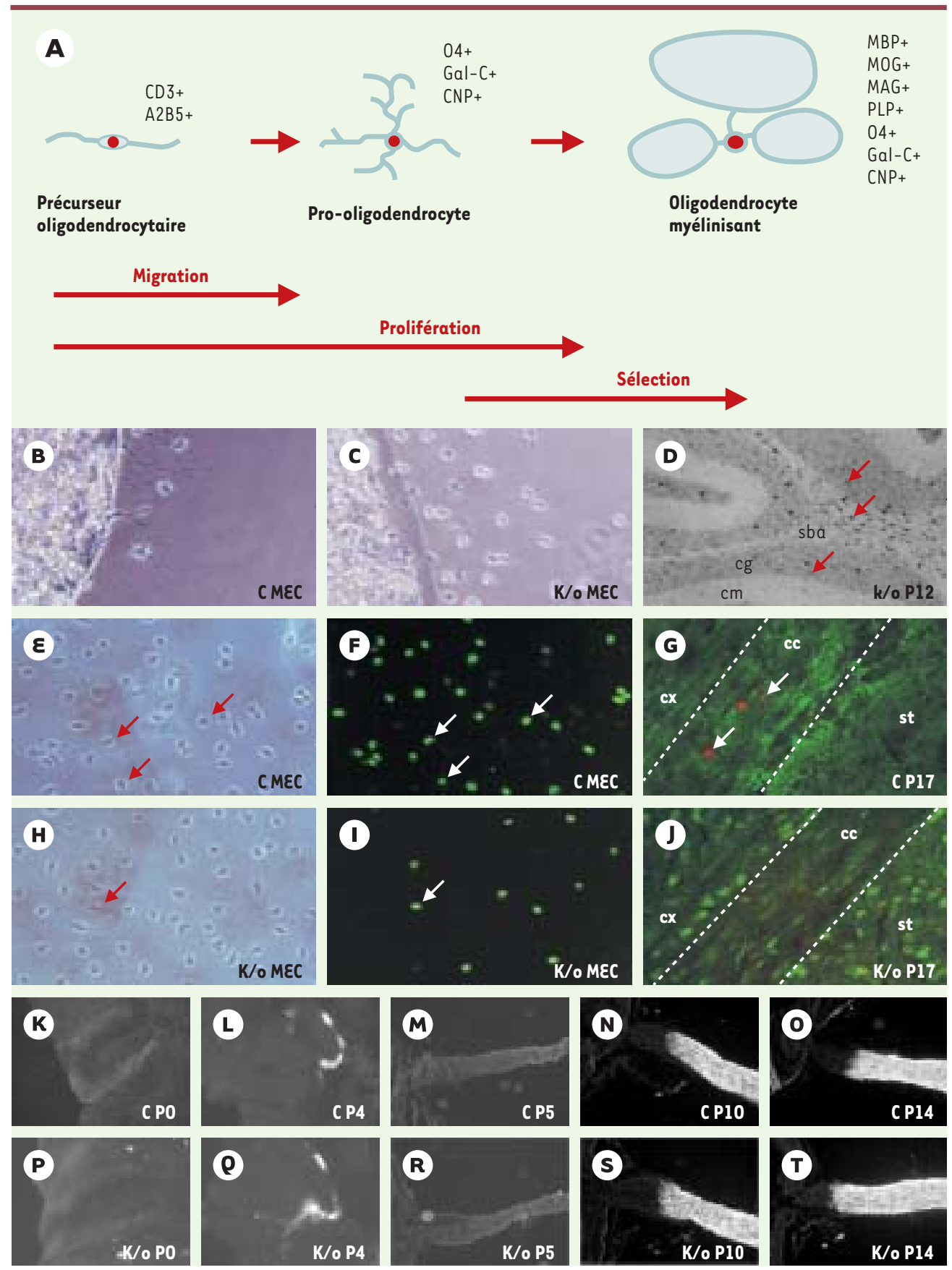

Figure 2. Implication fonctionnelle de la TN-C dans le comportement des cellules du lignage oligodendrocytaire. $A$. Schéma de la différenciation des progéniteurs oligodendrocytaires (PO) reconnue par l'expression de divers marqueurs phénotypiques. B-C. Agarose drop assay : migration des PO à partir d'une goutte d'agarose déposée sur une matrice astrocytaire après 24 heures : ( $M E C$ ) matrice astrocytaire témoin contenant la TN-C, ( $k / o M E C)$ matrice astrocytaire déficiente en TN-C. D. Absence de pénétration des PO dans la couche moléculaire $(\mathrm{cm})$ du cervelet au contraire de la couche granulaire (cg) et de la substance blanche adjacente (sba) au jour 12 post-natal (hybridation in situ du récepteur $\alpha$ du PDGF, flèches). $\varepsilon-F, H-I$. Défaut de prolifération des PO sur k/o MEC en comparaison à C MEC : $\boldsymbol{\varepsilon}$, $\boldsymbol{H}$ contraste de phase; $\boldsymbol{F}$, I. immunodétection de l'incorporation de bromodésoxyuridine 24 h après ensemencement. $G$, J. Apoptose réduite dans le corps calleux (cc) des souris déficientes en TN-C (méthode TUNEL, flèches), (cx : cortex, st : striatum). $K-T$. Profil et cinétique de myélinisation normale chez les souris déficientes en TN-C mis en évidence par immunomarquage de la MBP (myelin binding protein) dans le cervelet (K-L, P- $\rho)$ et dans le nerf optique $(M-O, R-T)$. 
augmente la prolifération des précurseurs oligodendrocytaires in vivo et in vitro (Figure $2 \varepsilon, F, H, I$ ). L'intégrine $\alpha v \beta 3$ étant le récepteur directement impliqué dans cet effet, après son interaction avec le récepteur $\alpha \mathrm{du}$ facteur de croissance dérivé des plaquettes (PDGF) (Figure 3) [22]. De telles interactions entre la TN-C et les signaux émanant des facteurs de croissance sont documentés dans d'autres systèmes biologiques [1] et nos résultats récents démontrent que la TN-C joue également un rôle essentiel dans les capacités de prolifération et de différenciation des cellules souches pluripotentes du SNC impliquant le bFGF, l'EGF et les bone morphogenetic proteins (BMP). Enfin, nous avons pu déterminer que la diminution de la prolifération des précurseurs oligodendrocytaires chez les souris déficientes en TN-C peut être contrebalancée par des mécanismes de corrections cellulaires (distinct de compensations moléculaires) en décelant une réduction de la mort cellulaire lors du processus de sélection dans des structures myélinisées telles que le corps calleux (Figure 2 G$H$, Figure 3) [22]. II est possible que ces mécanismes correcteurs expliquent de nombreux phénotypes apparemment normaux de souris transgéniques. Ils pourraient néanmoins s'avérer inefficaces lors de perturbations environnementales (lésions, cancers) au cours desquelles la molécule d'intérêt est ordinairement exprimée.

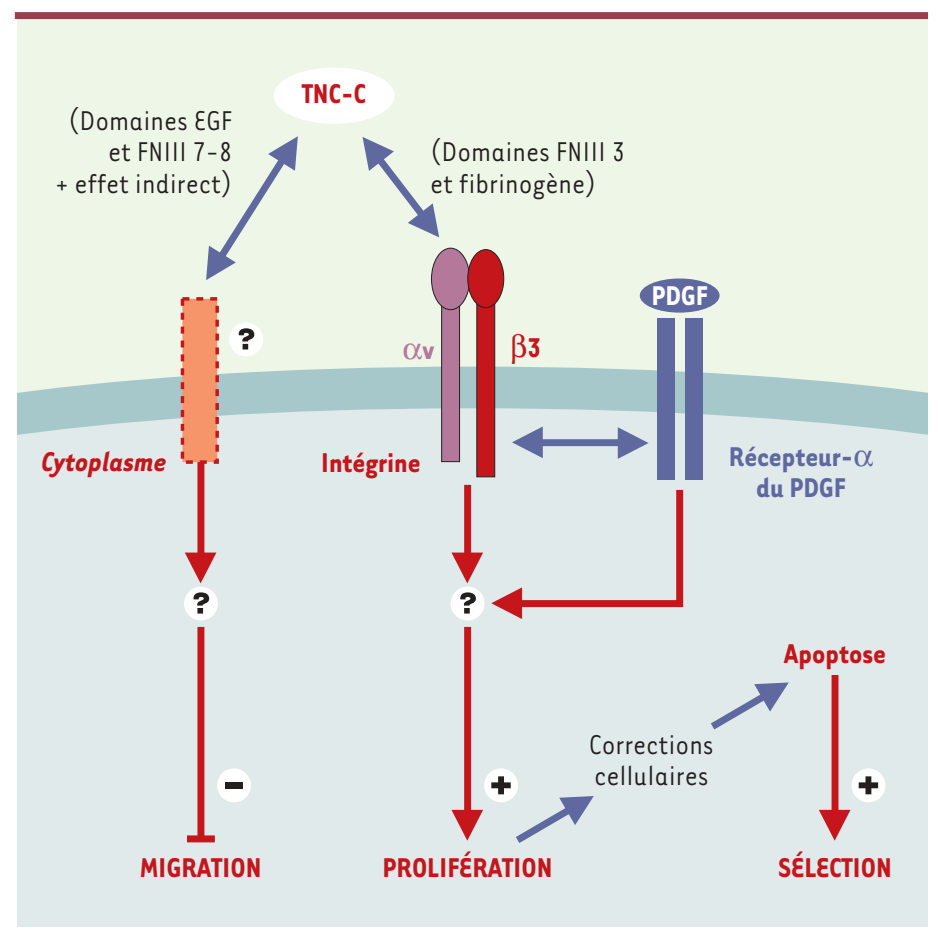

Figure 3. Modélisation des voies de signalisation impliquant la TN-C dans le comportement des précurseurs oligodendrocytaires.

\section{Conclusions}

Au vu de l'ensemble de ces données, il apparaît évident que la TN-C est impliquée dans divers aspects du développement du SNC. Ses fonctions soulignent particulièrement l'importance des interactions entre la matrice extracellulaire et le SNC, ainsi qu'entre les intégrines et les facteurs de croissance, dans la régulation du comportement des précurseurs du SNC. Les analyses détaillées de souris dont le gène codant pour la TN-C a été invalidé ont démontré l'existence de phénotypes parfois subtils et minutieusement corrigés au cours du développement, n'excluant cependant pas la présence de molécules constitutivement redondantes de la TN-C ou induites pour compenser son absence. La production de double-mutants pour deux molécules de la famille des ténascines ou d'invalidations conditionnelles pourra peut-être permettre de répondre à ces questions. Toutefois, des résultats récents obtenus en dehors du SNC, comme au cours de glomérulonéphrites, de l'hématopoïèse et de cancers mammaires [15], élargissent encore l'étendue du phénotype des souris dont le gène codant pour la TN-C a été invalidé et permettent en partie d'expliquer pourquoi cette glycoprotéine est génétiquement très conservée à travers les espèces. Enfin, de nombreux récepteurs et voies de signalisation restent encore à identifier, et l'avenir de la recherche sur les glycoprotéines de la matrice extracellulaire (actuellement en plein essor) réserve probablement quelques déceptions mais aussi quelques bonnes surprises qui pourront déboucher sur des applications médicales importantes comme ce pourrait être le cas dans la régénérescence axonale ou dans l'immunothérapie des gliomes. $\diamond$

\section{REMERCIEMENTS}

Le travail décrit dans cet article a été en partie financé par la Multiple Sclerosis Society of Great Britain and Northern Ireland et par le Wellcome Trust. 


\section{SUMMARY}

Tenascin-C:

an extracellular matrix molecule involved

in central nervous system development

Composed of a network of different collagens, proteo-

glycans and glycoproteins, the extracellular matrix constitutes an essential substrate for tissular morphogenesis and provides support and flexibility to mature tissues. Moreover, it acts as an informational epigenetic entity in that it transduces and integrates extracellular signals from various receptors of the plasma membrane. Among the extracellular matrix molecules which are expressed during central nervous system (CNS) develop- ment, tenascin-C (TN-C) has a very singular pattern of expression based on its spatio-temporal distribution and synthesised isoforms. Numerous in vitro studies suggested specific roles for TN-C in CNS development, notably in neural precursor cells migration as well as in neurite guidance and outgrowth. Also, the production of TN-C null mutant, initially described without gross structural abnormalities, allowed to investigate in more details and in vivo the potential involvement of this glycoprotein in CNS ontogenesis. Recent works tend to emphasize ancient concepts but also to reveal new functions such as in the proliferation of oligodendrocyte precursor cells. $\nabla$

\section{RÉFÉRENCES}

1. Jones FS, Jones PL. The tenascin family of ECM glycoproteins : structure, function and regulation during embryonnic development and tissue remodeling. Dev Dyn 2000 ; $218: 235-59$

2. Erickson HP, Inglesias JL. A six-armed oligomer isolated from cell surface fibronectin preparations. Nature 1984 ; 311 : 267-9.

3. Joester A, Faissner A. Evidence for combinatorial variability of tenascin-C isoforms and developmental regulation in the mouse central nervous system. J Biol Chem 1999 ; 274 : 17144-51.

4. Joester A, Faissner A. The structure and function of tenascins in the nervous system. Matrix Biol 2001 ; $20: 13-22$.

5. Jones PL, Jones FS. Tenascin-C in development and disease : gene regulation and cell function. Matrix Biol 2001 ; 19: 581-96.

6. Crossin KL, Hoffman $S$, Grumet M, Thiery JP, Edelman GM. Site restricted expression of cytotactin during development of the chicken embryo. J Cell Biol 1986 ; 102 : 1917-30.

7. Kawano H, Ohyama K, Kawamura K, Nagatsu I. Migration of dopaminergic neurons in the embryonic mesencephalon of mice. Brain Res Dev Brain Res 1986; 86 : 101-13.

8. Kiernan BW, Götz B, Faissner A, ffrenchConstant C. Tenascin-C inhibits oligodendrocyte precursor cell migration by both adhesion-dependent and adhesion-independent mechanisms. Mol Cell Neurosci 1996 ; 7 : 322-35.

9. Faissner A, Kruse J. $\mathrm{Jl}$ /tenascin is a repulsive substrate for central nervous system neurons. Neuron 1990 ; 5 : 627-37.

10. Götz M, Scholze A, Clement $A$, et al. Tenascin-C contains distinct adhesive, anti-adhesive, and neurite outgrowth promoting sites for neurons. J Cell Biol 1996 ; 132 : 681-99.

11. Chuong CM, Crossin KL, Edelman GM. Sequential expression and differential function of multiple adhesion molecules during the formation of cerebellar cortical layers. J Cell Biol 1987 ; 104 : 331-42.

12. Götz M, Bolz J, Joester $A$, Faissner A. Tenascin- $C$ synthesis and influence on axonal growth during rat cortical development. EurJ Neurosci 1997 ; 9 : 496-506.

13. Saga Y, Yagi T, Ikawa Y, Sakakura T, Aizawa S. Mice develop normally without tenascin. Genes Dev 1992 ; 6: 1821-31.

14. Forsberg $\varepsilon$, Hirsch $\varepsilon$, Frohlich $L$, et al. Skin wounds and severe nerves heal normally in mice lacking tenascin-C. Proc Natl Acad Sci USA 1996 ; $93:$ 6594-9.

15. Kiernan BW, Garcion $\varepsilon$, Ferguson J, et al.. Myelination and behavior of tenascin-C null transgenic mice. Eur J Neurosci 1999 ; 11 : 3082-92.

16. Mackie $\varepsilon$ J, Tucker RP. The tenascin-C knockout revisited. J Cell Sci 1999 ; 112 : 3847-53.

17. Mège RM, Nicolet $M$, Pincon-Raymond M, Murawsky M, Rieger F. Cytotactin is involved in synaptogenesis during regeneration of the frog neuromuscular system. Dev Biol 1992 ; 149: 381-94.

18. Schachner M. Neural recognition molecules and synaptic plasticity. Curr Opin Cell Biol 1997 ; 9 : 627-34.

19. Cifuentes-Diaz C, Velasco $\varepsilon$, Meunier FA, et al. The peripheral nerve and the neuromuscular junction are affected in the tenascin-Cdeficient mouse. Cell Mol Biol 1998 ; 44 : 357-79.
20. Cifuentes-Diaz C, Faille L, Goudou D, Schachner M, Rieger F, Angaut-Petit D. Abnormal reinnervation of skeletal muscle in a tenascin-C-deficient mouse. J Neurosci Res 2002 ; 67 : 93-9.

21. Bartsch U. The extracellular matrix molecule tenascin$C$ : expression in vivo and functional characterization in vitro. Prog Neurobiol $1996 ; 49$ : 145-68.

22. Garcion $\varepsilon$, Faissner $A$, ffrench-Constant $C$. Knockout mice reveal a contribution of the extracellular matrix molecule tenascin- $\mathrm{C}$ to neural precursor proliferation and migration. Development $2001 ; 126: 2485-96$

23. Frost $\varepsilon$, Kiernan BW, Faissner A, ffrenchConstant C. Regulation of oligodendrocyte precursor migration by extracellular matrix : evidence for substrate-specific inhibition of migration by tenascin-C. Dev Neurosci 1996 ; 18 : 266-73.

\section{TIRÉS À PART}

દ. Garcion 